\title{
Features of the Soft X-ray Background and Implications for the EUV Background
}

\author{
C. R. BARBER AND R. S. WARWICK \\ Department of Physics and Astronomy, University of Leicester, \\ University Road, Leicester, LE17RH, UK
}

\begin{abstract}
A series of eight overlapping ROSAT PSPC observations situated in the Lockman hole region have been analysed in the $0.1-0.4 \mathrm{keV}$ band. The total exposure of $\sim 137 \mathrm{ks}$ and the ultra-low interstellar column density make the data extremely suitable for studies of the diffuse signal. After excluding the contribution of bright discrete sources the remaining signal was examined on a scale of $20^{\prime}$. As expected the $0.1-0.4 \mathrm{keV}$ signal exhibits a strong anticorrelation with column density. However, there is also evidence for significant excess fluctuations superimposed on this anticorrelation which may be attributable to some form of cloud structures within the hot ISM.

The diffuse signal expected in the ROSAT WFC all-sky survey for this region is predicted and shown to be far in excess of that observed. The origin of the fluctuations in the soft X-ray background and their possible relevance to the Extreme-Ultraviolet background are discussed.
\end{abstract}

\section{Introduction}

In the 30 years since the discovery of the X-ray background (XRB) considerable advances have been made in our understanding of this intriguing source of diffuse radiation. However, even with the enormous observational progress that has been made a comprehensive description of its origin remains a far distant prospect.

At energies $>2 \mathrm{keV}$ the diffuse emission of the XRB is without doubt of extragalactic origin. However, at lower energies, at least one additional component emerges and dominates the emission. In the $0.1-0.4 \mathrm{keV}$ band, where this work concentrates, the diffuse intensity is a factor $\geq 3$ above an extrapolation of the high energy power law and anticorrelates with galactic $N_{\mathrm{H}}$. All-sky maps of the diffuse emission, in well separated energy channels within the $0.1-0.4 \mathrm{keV}$ band, exhibit strong spatial intensity correlations (e.g. McCammon \& Sanders, 1990). As the absorbing cross section changes considerably across the $0.1-0.4 \mathrm{keV}$ range this is strong evidence for the emission originating in front of the majority of absorbing material.

Studies of the interstellar medium (ISM) using observations of the hydrogen Lyman series, interstellar absorption lines and Extreme-Ultraviolet (EUV) absorption have shown that the solar system is located near the center of a low-density cavity. A model has therefore been developed to account for the $0.1-0.4 \mathrm{keV}$ emission in which the local cavity is filled with an $\mathrm{X}$-ray emitting plasma. The observed anticorrelation between $\mathrm{X}$-ray intensity and $N_{\mathrm{H}}$ is then reproduced by a displacement mechanism; as the plasma extends further into the HI layer so the $N_{\mathrm{H}}$ reduces, the plasma emitting path length increases, and with it the observed $\mathrm{X}$-ray intensity. The emissivity inferred for the cavity from this model is consistent with a $10^{6} \mathrm{~K}$ gas at an acceptable interstellar pressure.

The aim of the work presented here is to test over a small area of sky the anticorrelation between $N_{\mathrm{H}}$ and $0.1-0.4 \mathrm{keV} \mathrm{X}$-rays, to decompose the soft X-ray signal into its constituents and to predict the contribution to the EUV background. To this end a series of eight overlapping ROSAT PSPC observations are employed (see Figure 1). They subtend $\sim 14$ degree $^{2}$ and have a cumulative exposure of $137 \mathrm{ks}$. High quality HI data for the region (kindly provided by F.J. Lockman) show that the north-south strip of 
observations, the "UK Medium Sensitivity Survey", span a region of relatively uniform HI $\left(N_{\mathbf{H}}=5.6-9.3 \times 10^{19} \mathrm{~cm}^{-2}\right)$. In contrast, the two observations which extend to the north-east, the "Lockman Spur" pointings, follow a clear HI enhancement (maximum $N_{\mathrm{H}}=14.0 \times 10^{19} \mathrm{~cm}^{-2}$ ).

\section{ROSAT PSPC Data Reduction}

Isolation of the diffuse soft XRB signal in a ROSAT PSPC dataset requires the careful removal of non-cosmic contaminants. Thus data has been rejected if taken at a time when the charged-particle detection rate was high. Similarly, only data accumulated during periods when the satellite was in earth shadow has been accepted. The light curves of the remaining data have been examined and all appear highly stable. This stringent process reduced the cumulative exposure of all 8 observations to $63.5 \mathrm{ks}$.

The $0.1-0.4 \mathrm{keV}$ band corresponds to ROSAT PSPC pulse invariant channels 11-40 and images have been cast in this range for use in the remainder of this analysis. Exposure and vignetting corrections have been performed using the flat-fielding technique described by Snowden et al. (1993). As the long and short term enhancement contaminants would not necessarily appear in the light curves there remains the possibility of an offset intensity between adjacent observations. This has been corrected by applying relative field-to-field offsets which reduce the overall baseline to the minimum recorded in the whole set of observations. The resulting images have been spatially "mosaiced" together for further analysis.

In order to reduce the discrete source contribution to the diffuse signal the mosaiced image has been searched for sources. The detection algorithm takes into account the varying background signal and detects sources brighter than $10^{-2}$ count $\mathrm{s}^{-1}$. Assuming a power law of energy index $\alpha=1$ and $N_{\mathrm{H}}=5.7-12.5 \times 10^{19} \mathrm{~cm}^{-2}$ this count rate corresponds to $0.5-2 \mathrm{keV}$ fluxes of $6.4-10.4 \times 10^{-14} \mathrm{erg} \mathrm{cm}^{-2} \mathrm{~s}^{-1}$. A mask is created which when applied to the raw mosaiced image masks out at least $95 \%$ of the flux from each detected source (the remaining surface brightness is insignificant). To produce a map of the residual diffuse emission (Figure 1) the raw mosaiced image is convolved with a $20^{\prime} \times 20^{\prime}$ top hat (the resolution of the pixels used in the remainder of this analysis), ignoring the masked regions. For statistical analysis a sample of 97 independent pixels are extracted from this map excluding at this stage those coincident with Abell clusters. The mean count rate in a pixel is $947 \pm 65.6 \times 10^{-6}$ count s$^{-1} \operatorname{arcmin}^{-2}$. There is clearly considerable variation in the observed count rate, a significant proportion of which is caused by a general anticorrelation with $N_{\mathrm{H}}$.

\section{Examination of the Diffuse $0.1-0.4 \mathrm{keV}$ Band Signal}

Considering the diffuse signal in each individual field there are two potential sources of intrinsic scatter namely counting statistics and confusion noise. For a typical exposure and count rate the former induces a scatter of $\sim 2 \%$ in the diffuse intensity measured in a pixel. The latter can be estimated as the distribution in flux per unit solid angle of discrete sources is known (Hasinger et al. 1993); simulations show that the typical scatter caused by this effect is $\sim 2.8 \%$ of the total diffuse intensity for the pixel size used here. Monte Carlo simulations including both of these effects yield a predicted rms scatter between the pixels in any individual field of $37 \times 10^{-6}$ count s ${ }^{-1}$ arcmin $^{-2}$. Table 1 shows the mean count rate and scatter observed in each field. It is clear that for seven of the eight observations the scatter is considerably more than expected from just counting statistics and confusion noise. 
TABLE 1. Mean, scatter and standard error $\left(\times 10^{-6}\right.$ count s $\left.\mathrm{s}^{-1} \operatorname{arcmin}^{-2}\right)$ for each field.

$\begin{array}{ccc}\text { Field } & \text { Mean } & \text { Standard error } \\ \text { UKMS 1 } & 998 \pm 34.8 & 9.3 \\ \text { UKMS 2 } & 917 \pm 44.3 & 12.3 \\ \text { UKMS 3 } & 939 \pm 43.6 & 10.6 \\ \text { UKMS 4 } & 982 \pm 49.5 & 11.4 \\ \text { UKMS 5 } & 983 \pm 64.3 & 15.6 \\ \text { UKMS 6 } & 905 \pm 51.5 & 21.0 \\ \text { SPUR 1 } & 949 \pm 51.1 & 11.4 \\ \text { SPUR 2 } & 875 \pm 64.5 & 16.1\end{array}$

Probing field-to-field diffuse signal fluctuations requires consideration of the scatter induced by the field-to-field correction process. This is typically $15 \times 10^{-6}$ count s s $^{-1}$ $\operatorname{arcmin}^{-2}$. Combining this scatter with the standard errors given in Table 1 does not account for the observed field-to-field variation implying that there are also significant fluctuations present on this scale.

Comparing the HI map for the region and the $0.1-0.4 \mathrm{keV}$ diffuse signal map (Figure 2) shows that a significant part of the non-statistical scatter highlighted above arises from the general anticorrelation between $N_{\mathrm{H}}$ and X-ray intensity. To explore this further the data have been fit with an absorption model which assumes spatially uniform foreground and background intensities, the latter being subject to varying attenuation dictated by the line-of-sight $N_{\mathbf{H}}$. (The absorption model is chosen rather than a displacement model as Snowden et al. (1991) and Burrows \& Mendenhall (1991) have shown that in some directions a large fraction of the soft X-ray background originates well beyond the local cavity. Further the quality of the fit is relatively independent of the assumed model.) In this case all eight fields are considered together and the error bars are as measured from the pixels and include the scatter caused by the field-to-field correction process. The best fit has $\chi_{v}^{2}=1.5$, a local component of $646 \pm 70 \times 10^{-6}$ count s$^{-1}$ arcmin $^{-2}$ and a distant component of $675 \pm 159 \times 10^{-6}$ count s$^{-1}$ arcmin $^{-2}$ (where the errors are for $90 \%$ confidence). The rms scatter about the predicted model is $52 \times 10^{-6}$ count s$^{-1} \operatorname{arcmin}^{-2}$; considerably greater than the $40 \times 10^{-6}$ count s$^{-1} \operatorname{arcmin}^{-2}$ predicted by our simulations. An image of the observed diffuse count rate with the best fit absorption model subtracted is shown if Figure 2 and is characterised by many deviations to $\pm 100 \times 10^{-6}$ count s s $^{-1}$ $\operatorname{arcmin}^{-2}$. Further, these deviations extend over scales $>20^{\prime}$ and appear to form coherent structures.

\section{Implications for the Diffuse EUV Background Signal}

Having isolated the $0.1-0.4 \mathrm{keV}$ diffuse signal the EUV diffuse signal can be predicted. For the purpose of this comparison the results from the ROSAT WFC S1a filter are utilised (see West et al., these proceedings). In the energy range of the Sla filter an $N_{\mathbf{H}}$ of $8 \times 10^{19} \mathrm{~cm}^{-2}$ presents seven optical depths to a $10^{6} \mathrm{~K}$ thermal plasma spectrum. Therefore the distant component derived in fitting the absorption model to the $0.1-$ $0.4 \mathrm{keV}$ band data above can be ignored. Folding the derived foreground count rate through the relevant responses using a Raymond \& Smith $10^{6} \mathrm{~K}$ plasma model spectrum (Raymond \& Smith 1977, Raymond 1991 privately communicated computer code update) yields a predicted S1a count rate of 2.7 count $\mathrm{s}^{-1}$ across the WFC field-of-view. West et al. however, derive a $99 \%$ upper limit of 0.5 count $\mathrm{s}^{-1}$. 
Data from the Diffuse X-ray Spectrometer (Sanders, these proceedings) has shown that current thermal plasma models, such as that used here, fail to fit high resolution $150-284 \mathrm{eV}$ spectra (even with a variety of assumed elemental depletions). The analysis presented here illustrates a similar failure of the models but over a considerably wider energy range. It is clear that there are fundamental flaws in our assumptions about the state of the plasma that is responsible for the soft X-ray background. Detailed fitting of high quality spectra and a comprehensive understanding of the physics in the plasma are required to resolve this conundrum.

This analysis has revealed an important feature of the morphology of the soft $\mathrm{X}$ ray background - the existence of small scale fluctuations superimposed on the general anticorrelation with $N_{\mathrm{H}}$. The ROSAT observations imply that a typical fluctuation has a magnitude of $\sim 30 \times 10^{-6}$ count $\mathrm{s}^{-1} \operatorname{arcmin}^{-2}$. It is likely that these fluctuations are caused by clumping of the hot ISM along the line-of-sight. To illustrate the physical conditions of this plasma assume that it is contained within a spherical cloud. Given the count rate of the fluctuation and the temperature of the plasma the emission measure, $\int n_{e}^{2} \mathrm{~d} l$, of the plasma in the cloud can be computed. The emitting path length, $\mathrm{d} l$, is limited by the angle subtended by the cloud, (taken to be $20^{\prime}$ ), and therefore the electron density, $n_{e}$ and thermal pressure, $n_{e} T$, can be derived. Cox \& Reynolds (1987) have estimated that hot X-ray emitting plasma in the ISM has a pressure budget $10^{4} \mathrm{~cm}^{-3} \mathrm{~K}$. (In this analysis an acceptable range of $0.5-1.5 \times 10^{4} \mathrm{~cm}^{-3} \mathrm{~K}$ is assumed.) Therefore acceptable distances to the cloud can be determined. If the cloud is placed in front of galactic $N_{\mathrm{H}}$ then its distance is constrained to $150-500 \mathrm{pc}$. Alternatively if the cloud is beyond the galactic $N_{\mathrm{H}}$ then it is constrained to a distance $500-3000 \mathrm{pc}$. A further cloud diagnostic is their predicted lifetime which can be estimated using the adiabatic sound crossing time. For the cloud in front of the galactic $N_{\mathrm{H}}$ the lifetime is $2 \times 10^{4}-7 \times 10^{4}$ years and for the cloud behind the galactic $N_{\mathrm{H}}$ it is $3 \times 10^{4}-1 \times 10^{6}$ years.

Currently it is unclear as to whether the fluctuations arise within the local cavity or beyond. Further work is being undertaken to characterise the angular scales of the fluctuations. Also a large sample of ROSAT PSPC pointings are being examined to determine the amplitude of the fluctuations as a function of galactic $N_{\mathrm{H}}$. It is possible that if the fluctuations are generated locally then the local plasma arises not from the cooling of an elderly supernova remnant, but rather from a more vigorous phase of its existence. If this is the case then the fluctuations represent a morphological constraint that will have to be satisfied by models which produce spectra of the sort observed by the DXS. If, however, the fluctuations have a distant origin then they may represent significant evidence for circulatory models of the ISM.

\section{REFERENCES}

BurRows, D. \& Mendenhall, J. 1991, Nature, 351, 629

Cox, D. \& REYNOLDS, R. 1987, ARA\&A, 25, 303

Hasinger, G., Burg, R., Giacconi, R., Hartner, G., Schmmt, M., Trumper, J. \& ZAMORANI, G. 1993, A\&A, 275, 1

McCAMmon, D. \& SANDERS, W. 1990, ARA\&A, 28, 657

RAYMOND, J. \& SMITH, B. 1977, ApJS, 35, 419

Snowden, S., McCammon, D., Burrows, D. \& Mendenhall, J. 1993, ApJ, 28, 657

SNOWDEN, S. ET AL. 1991, Science, 252, 1529 